\title{
Una aproximación sociocultural a las formas de regulación emocional en cuidadores familiares de enfermos crónicos en Guadalajara, Jalisco
}

\author{
Elba Karina Vázquez Garnica \\ y María del Rocío Enríquez Rosas
}

La investigación buscó la comprensión de las formas de regulación de las emociones de familiares cuidadores de enfermos crónicos a partir de la perspectiva del construccionismo social moderado. El método utilizado fue una etnografía profunda basada en la observación y en entrevistas cualitativas semiestructuradas a 19 cuidadores. Los datos muestran la relevancia religiosa y espiritual para la regulación de las emociones en los discursos y prácticas cotidianas de los cuidadores familiares. El estudio enriquece los planteamientos de Hochschild (1990) y expone la existencia de un modelo con dos funciones: el manejo de la situación inmediata y el bienestar del enfermo y del cuidador con énfasis en la dimensión relacional. El cuidado como objeto de estudio a partir de perspectivas interdisciplinarias coloca a lo sociocultural en el centro de la discusión.

PALABRAS CLAVE: cuidador, emociones sociales, regulación emocional, etnografía de las emociones, enfermedad crónica

\section{A Sociocultural Approach to the Emotional Regulation that Family Caregivers Enact upon Chronic Patients in Guadalajara, Jalisco}

The research aimed to understand the emotional management forms displayed by family caregivers of chronic patients from the moderate social constructionism perspective. The methods used were ethnography, observation and qualitative semi-structured interviews with 19 caregivers. The data show relevant religious and spiritual content within discourses and everyday practices. This study enriches the Hochschild (1990) postulates and reveal the existence of a double function model: the resolution of the immediate situation and the well being of the patient and the caregiver with emphasis on the relational dimension. The relevance of socio-cultural elements takes centrality when approaching care as an object of study from interdisciplinary perspectives.

KEYWORDS: caregiver, social emotions, emotional management, emotions ethnographic, chronic illness

\footnotetext{
Elba Karina Vázquez Garnica: Centro Universitario de Ciencias de la Salud, Universidad de Guadalajara,

Guadalajara, Jalisco, México

karinavazq@gmail.com

MARÍA DEL Rocío Enríquez Rosas: Instituto Tecnológico y de Estudios Superiores de Occidente,

Tlaquepaque, Jalisco, México

rocioe@iteso.mx
}

Desacatos, núm. 39, mayo-agosto 2012, pp. 57-72

Recepción: 9 de noviembre de 2011 / Aceptación: 6 de febrero de 2012 


\section{INTRODUCCIÓN}

$\mathrm{E}$ 1 cuidado de un familiar ha sido una práctica común desde siglos pasados entre la población dependiente: niños, discapacitados, ancianos o enfermos (Tronto, 1993). Se trata de una práctica social en la que predomina la precariedad, la vulnerabilidad social y un efecto importante en la salud de los cuidadores familiares. La mayoría de las veces son mujeres quienes realizan esta tarea $y$ quedan fuera del sistema de trabajo remunerado, del crecimiento profesional y económico. No obstante, el cuidado en el ámbito de la salud adquiere notoriedad de cara al envejecimiento y al aumento de enfermedades crónicas, según las proyecciones demográficas 2000-2050, periodo en que se contempla una proporción reducida de cuidadores en relación con la población dependiente (CEPAL, 2009). En Iberoamérica, el "cuidado" es estudiado desde paradigmas económicos (Vara, 2006, entre otros), de derecho (Pautassi, 2007, entre otros) y a en los acercamientos de corte cualitativo en el campo de la salud (Robles, 2007; Pinheiro y Araújo de Mattos, 2008 a y b, 2009; Pinheiro y Coelho Lopes, 2010; Enríquez, 2011). Sin embargo, la parte emocional y simbólica ha sido considerada de manera marginal, a pesar de que las emociones son parte intrínseca del cuidado.

La producción en torno al tema se concentra en el área de la salud, principalmente en disciplinas como la enfermería, la psicología y en especialidades médicas como la psiquiatría y la geriatría. En las ciencias sociales hay una producción menor, en trabajo social, sociología y antropología. Predominan los enfoques centrados en el estrés con resoluciones metodológicas cuantitativas (Vázquez, 2008). Estas aproximaciones teórico-metodológicas requieren de miradas cualitativas complementarias que favorezcan la comprensión integral de las formas y dinámicas de interacción de las emociones en relación con el cuidado, en tanto objeto de estudio interdisciplinar. Al ponderar la visión positivista se descuidan

los aspectos sociales de carácter subjetivo e intersubjetivo que coadyuvan a la construcción de las narrativas y de las prácticas socioculturales necesarias para el cuidado en el campo de la salud.

La veta a explorar tiene que ver con los aspectos socioculturales relativos al cuidado, específicamente respecto de la población de cuidadores/familiares de enfermos crónicos, sus emociones y estrategias de regulación emocional, tanto para protección personal como para el mantenimiento de la práctica del cuidado ante la precariedad de recursos materiales, sociales y simbólicos. Este trabajo ofrece conocimiento sobre un fenómeno estudiado de manera aislada en la producción científica mexicana, en la antropología, la sociología y la psicología social, y ayuda a comprehender la situación emocional, social y de salud de cuidadores de enfermos crónicos y las estrategias que despliegan para regular sus emociones de acuerdo con su contexto sociocultural y con la función de dichas estrategias.

En el estudio se consideraron la teoría feminista de la ética del cuidado (Ungerson, 1983; Graham, 1983; Tronto, 1993; Gilligan, 1995; Engster, 2005) y la teoría construccionista de la sociología de las emociones (Hochschild, 1990; Gordon, 1990; Enríquez, 2008), así como la antropología de las emociones (Le Breton, 1998). Además, se ofrece un panorama sociocultural del cuidado y de las estrategias de manejo emocional desde la perspectiva de un sujeto que cuida al otro. A continuación se describen los elementos teóricos que fueron fundamentales de la teoría feminista de la ética del cuidado.

a) La visión del cuidado como una construcción social dentro del sistema de trabajo y parte de las actividades de reproducción social. Cuidar es una acción subjetiva que se inscribe en una lógica social más amplia, misma que abarca principios sociales, políticos y económicos. En la interacción cuidadordependiente se implican elementos subjetivos - el sentimiento, la experiencia, lo simbólico- $\mathrm{y}$ aspectos socioculturales - la normas, los valores y las obligaciones- (Graham, 1983; Tronto, 1993). b) El cuidado es un trabajo físico y una experiencia de 
afecto que suponen cargas emocionales disímiles (Graham, 1983). Cuidar implica un trabajo emocional en el que el cuidador maneja sus sentimientos y coadyuva con los del dependiente (Ungerson, 1983). c) Cuidar es un intercambio interpersonal entre cuidador y dependiente, en el que la dependencia se explica a partir de las condiciones socioculturales que la generan: contexto social, ambiente sociourbano, políticas sociales. Es decir, lleva implícita la posición adquirida por el reconocimiento social.

Por otra parte, de la teoría construccionista de la sociología de las emociones se retomaron los lineamientos de Gordon (1990), que describe las emociones como elementos simbólicos subjetivos, producto de la interacción de las personas con su entorno sociocultural. Las tesis de Hochschild (1990) y Le Breton (1998) también explican la regulación emocional como acciones físicas y cognitivas que realizan las personas para mitigar $\mathrm{u}$ ocultar sus emociones. Los elementos considerados por el construccionismo social de las emociones para la regulación emocional son: a) La emoción y la regulación emocional son construidas en la interacción sociocultural. Dicho bagaje sociocultural está, a su vez, sujeto al momento sociohistórico en que están insertos los actores sociales (Gordon, 1990; Hochschild, 1990). b) El contexto sociocultural incluye las normas de regulación de las emociones a partir de las cuales las personas expresan o controlan su emoción, tomando en cuenta el escenario social en el que se desenvuelven (Gordon, 1990; Le Breton, 1998). c) La jerarquía en la relación interpersonal marca la referencia de la posición que tiene la persona respecto de los otros. De dicha jerarquía depende cómo y con quién los individuos regulan sus emociones y su expresión (Le Breton, 1998). Para el desarrollo del presente estudio se articularon los dos marcos teóricos: la teoría feminista de la ética del cuidado y la teoría construccionista de la sociología de las emociones. Juntas logran explicar la retroalimentación continua entre aspectos subjetivos e intersubjetivos y macrosociales, y permiten entender el cuidado y las estrategias de regulación emocional desde esta perspectiva. La pregunta que guió la investigación fue: ¿Cómo los cuidadores de enfermos crónicos regulan sus emociones vinculadas al trabajo de cuidado?

\section{METODOLOGÍA}

La selección de la población de estudio y de los informantes se llevó a cabo en instituciones de salud de tercer nivel de atención a las que acudieron los enfermos y sus cuidadores. En el Centro Médico Nacional de Occidente del Instituto Mexicano del Seguro Social (IMSs) se contactaron a cuidadores de enfermos de cáncer y diabetes con un nivel socioeconómico medio, en el Hospital Civil "Juan I. Menchaca" se localizaron a cuidadores de enfermos con diabetes y en la organización no gubernamental Albergue Mano Amiga se acercaron cuidadores de enfermos con cáncer, estos últimos de nivel socioeconómico bajo (INEGI, 1994). La participación de sujetos con un perfil socioeconómico diferenciado y los dos padecimientos - cáncer y diabetes - hizo posible la realización de ejercicios de contraste centrados en el foco de la investigación, que se refiere a las estrategias, en tanto prácticas, de regulación emocional.

La selección de los sujetos se realizó mediante muestreo teórico. Los cuidadores debieron reunir las siguientes características: ser familiar del enfermo con cáncer o diabetes mellitus tipo II, reconocerse a sí mismos y ser reconocidos por el enfermo como la persona responsable de atenderlo y acompañarlo, residir durante la etapa de la investigación en la Zona Metropolitana de Guadalajara, pertenecer a un estrato socioeconómico medio y bajo (INEGI, 1994) y aceptar participar en esta investigación. Se desplegaron cuatro estrategias para contactar a los informantes: 1) la invitación al estudio -el primer acercamiento con el cuidador y la invitación fueron simultáneas-; 2) bola de nieve, cuidadores que ya participaban en el estudio invitaron a otras personas en una situación similar a la suya; 3 ) la participación 
voluntaria, las personas se acercaron espontáneamente a platicar sus experiencias con la entrevistadora o pidieron ser incluidas en el estudio, y 4) el contacto por una tercera persona, uno de los profesionales de la salud de la institución que identificaba al cuidador del enfermo tomó sus datos y la entrevistadora contactó e invitó a los cuidadores al estudio.

Los datos se recolectaron mediante entrevistas en forma de conversaciones, basadas en un guión desarrollado de acuerdo con el relato biográfico (Pujadas, 2000). La observación y el registro riguroso de la entrevista ayudaron a entender las narrativas de los cuidadores según su entorno sociocultural y los espacios sociales en que acontece su vida cotidiana. Las entrevistas cualitativas y la observación sistemática permitieron rastrear y profundizar en las construcciones socioculturales del cuidado las emociones asociadas al mismo y las prácticas de regulación emocional (Hemmersley y Atkinson, 1994).

El procesamiento de los datos se hizo con base

\section{RESULTADOS}

En el estudio participaron 19 cuidadores, 16 mujeres $\mathrm{y}$ tres hombres. Las relaciones con el enfermo fueron: 11 hijos, cuatro cónyuges, dos hermanos, una nieta y una nuera. De cada institución participaron cinco personas, a excepción del Hospital Civil "Juan I. Menchaca", del que había cuatro representantes. Las características de los informantes por institución y grupo social fueron: 1) del Albergue Mano Amiga participaron cuatro mujeres y un hombre que oscilaban entre los 25 y 62 años de edad, cuya escolaridad máxima era de carrera técnica; 2) del Centro Médico Nacional de Occidente participaron cinco cuidadoras de enfermos con cáncer, todas mujeres, con edades entre los 25 y 52 años de edad, y escolaridad máxima de maestría y mínima de carrera técnica; 3) para el caso de informantes cuidadores de enfermos con diabetes participaron cuatro personas del Hospital Civil "Juan I. Menchaca", tres mujeres y un hombre, de entre 50 y 68 años de edad, con escolaridad máxima de primaria terminada y sin escolaridad, y 4) del Centro Médico Nacional de Occidente participaron cuatro mujeres y un hombre, de entre 16 y 57 años de edad, con escolaridad máxima de doctorado y mínima de primaria concluida. En general, los cuidadores recibieron apoyo económico de familiares, amigos e instituciones gubernamentales y no gubernamentales para sobrellevar los gastos de la atención a la enfermedad de su familiar.

\section{LA REGULACIÓN DE LAS EMOCIONES EN EL CUIDADOR: UNA MIRADA CUALITATIVA}

Para entender las estrategias de regulación emocional es menester identificar los aspectos socioculturales que los cuidadores mencionaron como fundamentales en sus roles y en el manejo de sus emociones. Los cuidadores narraron sus experiencias emocionales con elementos de su historia social, expresaron sus normas, valores y creencias, construidos con base en 
su interacción con otros sujetos sociales. Estos aspectos, aunados a la creencia en Dios, en los espíritus y en el destino, fueron cardinales en su regulación emocional. Los valores religiosos fueron traslapados a la vida familiar y del cuidado, independientemente de la situación socioeconómica o de la enfermedad del familiar, y coadyuvaron a mantener a la persona en el rol del cuidador, en el caso de cuidadores hijos, la idea de ser buen o mal cristiano y los preceptos de honrar a su padre y a su madre. Las costumbres familiares también jugaron un papel sustancial en la regulación de las emociones, como había sido reportado (Gordon, 1990). Gardenia narró su historia:

Mi mamá era de que tenía las cosas [comida], pero lo que te daba y ya, lo demás no tenías que agarrar. Entonces, yo así estaba acostumbrada y ya cuando empecé a trabajar pos ahí era de que lo que yo quería, yo podía agarrar... En mi casa era de si quería más, por decir algo fruta, naranjas, pos de una naranja, y si quería otra, pos ya no, porque esas eran digamos para mañana o para el niño más chico, eso cuando hay poco. Pero te digo, yo eso lo miraba como algo normal. Entonces, era lo mismo. Con el afecto igual: a uno en su casa nunca lo acostumbraron a eso del abrazo y el beso (Gardenia, 2009).

Gardenia compara su situación socioeconómica con su historia de afecto en la familia: la limitación de alimento y de cariño. En su narración se identifica cómo la cultura social y familiar es transmitida a través de las costumbres y crea pautas de convivencia y de organización familiar. Estos modelos son parte de la historia personal del cuidador y posteriormente se reproducen en la relación de cuidado. Gardenia fue una de las cuidadoras que se limitó a desarrollar las tareas instrumentales del cuidado, sin ningún tipo de acercamiento afectivo con su madre enferma. Otros factores, como ser el hermano menor, ser mujer u hombre, tener la etiqueta social de enojón, fuerte, chillón o sensible, contribuyeron en las expresiones emocionales y en las acciones de regulación. Los cuidadores hicieron una discriminación de fronteras borrosas entre elementos personales y sociales para identificar cómo expresar o no su emoción, de acuerdo con el sujeto con quien interactuaron y el espacio social. Cuando la persona adquiere el rol de cuidador cuenta con un bagaje cultural, social, familiar y personal que sirve de base para poner en práctica las estrategias de manejo emocional aprendidas e incluso para implementar otras de acuerdo con la nueva situación emocional, como el proceso de cuidado.

\section{Estrategias de regulación emocional ante el cuidado del otro: alcances y limitaciones}

En su investigación con asistentes de vuelo, Hochschild (1990) precisó dos métodos de manejo emocional. El primero definido como superficial, en el que se insertaron la no expresión emocional o la expresión de una emoción diferente. El segundo llamado profundo, conformado por dos tipos de acciones: por un lado, el control de sensaciones corporales y distanciamiento de la situación; por otro, fragmentar la situación al centrar la atención en un punto neutro, de manera que la emoción queda sujeta al cambio de la atención. Con base en las aportaciones de Hochschild (1990) y en el análisis del material empírico, se observó que las estrategias de regulación emocional en los cuidadores de enfermos crónicos con cáncer y diabetes fueron más complejas que las descritas por la autora. La categorización de superficiales o profundas no fue aplicable en el caso de los cuidadores, porque se identificaron siete funciones primarias en las estrategias de regulación emocional.

La función inicial de contener es controlar la emoción y las sensaciones corporales a nivel interno. Simular, otra de las funciones básicas, tiene como propósito el control sobre la expresión emocional, la restricción comunicativa. Las estrategias orientadas a la contención estuvieron vinculadas con las 


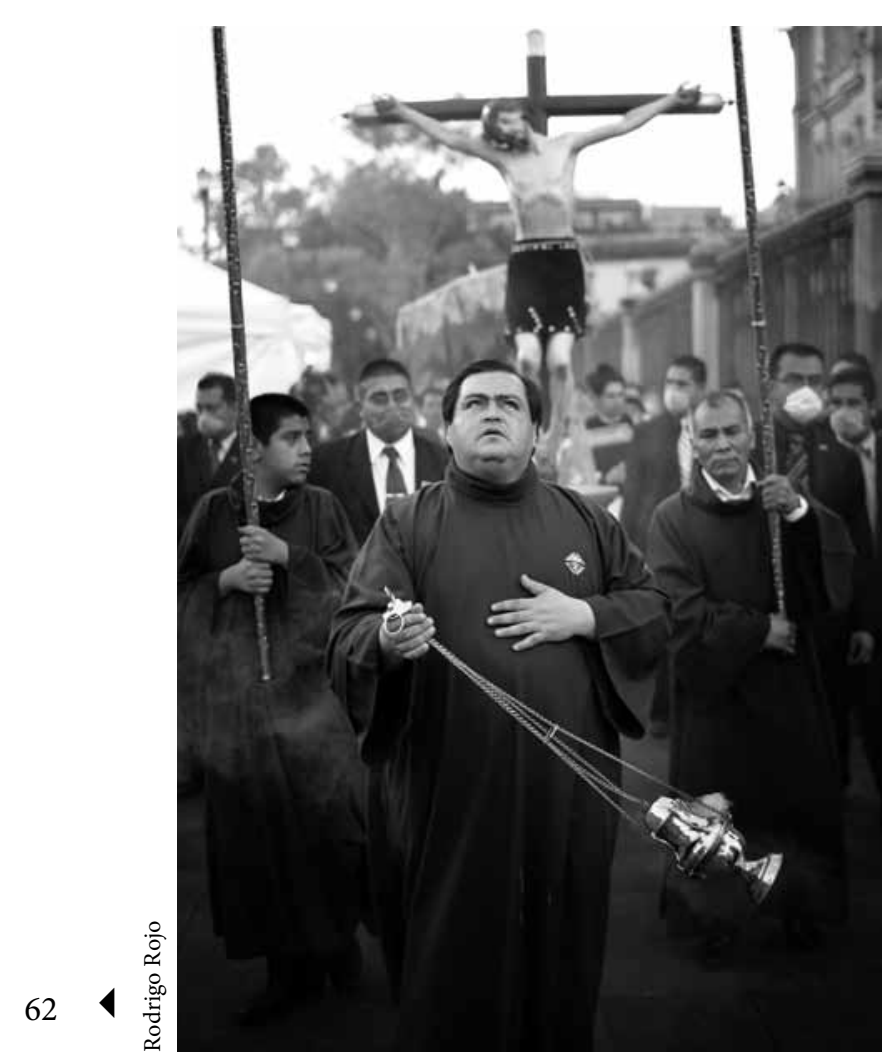

Procesión con el Cristo de la Salud por la erradicación de la influenza en la Catedral Metropolitana, 2009.

de simulación. Sin la capacidad de contener las emociones y las sensaciones corporales no era posible llevar a cabo la simulación en la expresión verbal y gestual. Con el propósito de lograr la contención emocional, los cuidadores procuraron no hablar y retirarse al sentir enojo para evitar discutir con el enfermo. Cuando se sintieron dispuestos, reanudaron la interacción. Otra de las estrategias para contener la emoción fue evadir la mirada, es decir, evitar el contacto ocular para salvaguardar la emoción para sí mismos y mantener el estatus de la relación en la fortaleza del cuidador respecto del enfermo.

Los cuidadores también usaron el lenguaje metafórico para comentar que deben "tragarse la emoción" frente al enfermo, para no evidenciar contenidos afectivos con posible connotación negativa sobre la enfermedad o las actitudes de los profesionales de la salud, o bien ante la incomprensión social por la situación de discapacidad del familiar enfermo. El acto simbólico de tragarse la emoción significó la falta de procesamiento de la experiencia emocional y una forma de contención durante diferentes periodos - desde algunas horas hasta días o semanasquellevaron a los cuidadores a evitar la confrontación de un orden social preestablecido a partir de reglas implícitas sobre las formas, los discursos y las prácticas del cuidado del otro, en este caso de un familiar cercano.

No llorar, cambiar de tema y expresar una emoción diferente a la que se siente fueron otras formas de contener y simular. Los cuidadores comentaron que lo hacen para no dar a conocer sus emociones y como una alternativa de protección hacia el enfermo y de esta manera, más o menos consciente, favorecer el mantenimiento y reproducción de formas convencionales del cuidado social, que requieren ser cuestionadas y transformadas con miras a un cuidado cada vez más social, colectivo y complementario entre los actores sociales. Desde la perspectiva de los cuidadores, el llanto y la tristeza estuvieron relacionados con debilidad y sensibilidad. En este sentido, reportaron preferencia por aquellas prácticas que favorecieran formas múltiples para ocultar el sufrimiento. Además, refirieron la expresión del enojo como más viable por su relación con la fortaleza más que las emociones asociadas con la tristeza y el dolor. Las acciones para contener y simular se dirigieron principalmente a la tristeza, el dolor o sentimientos vinculados con la actitud del enfermo hacia el tratamiento, la enfermedad o el cuidador, el nivel de evolución de la enfermedad, la situación del cuidado en general y la relación con el enfermo.

La tercera función básica fue evadir. El medio principal que los cuidadores usaron para lograrlo fue la distracción: realizaron cualquier actividad ajena a la situación del cuidado. Centraron su atención en algo diferente que les permitiera no sentir de forma momentánea o definitiva la emoción. Estar con otros fue una opción para distraerse del 
trabajo cotidiano de cuidado o para manejar la frustración que sintieron respecto de su situación económica y de las emociones relacionadas con la expectativa de la enfermedad de su familiar. Salir a caminar ayudó ante el estrés, la tristeza o la angustia producidas por las actividades cotidianas del trabajo de cuidado. Esta actividad fue considerada como un momento para estar a solas y no pensar en la situación emocional, distraerse y relacionarse con otras personas ajenas a la situación de cuidado. Bailar ayudó a evadir el nerviosismo y el estrés que provoca la monotonía del quehacer del cuidado y las situaciones emocionales surgidas en ese contexto. Escuchar música y ver televisión fueron estrategias recurrentes cuando los cuidadores estuvieron tristes, desesperados por la situación del enfermo y su actitud ante la enfermedad y cuando extrañaban sus actividades de antes de ser cuidadores, también cuando vivieron el nerviosismo, el enfado y la angustia por la rutina del cuidado y por la incertidumbre.

Retirarse de la situación específica de cuidado fue otra de las estrategias para evadir contenidos emocionales. Esto ayudó ante el coraje, el estrés, la desesperación, la molestia, la impotencia y los conflictos familiares asociados con el cuidado de un pariente enfermo y con las actitudes de este último, como reclamos hacia el cuidador o bien la falta de colaboración y adherencia al tratamiento. Los hallazgos coinciden con Stalberg, Ekerwald y Hultman (2004) respecto de la estrategia de retirarse de la situación como medio para afrontar sentimientos de confusión, tristeza, enojo y frustración, aunque en ocasiones favoreció que se evadiera la emoción de manera pasajera. Algunos cuidadores sintieron de nuevo la emoción al terminar la serie de estrategias de evasión. Esto demuestra que retirarse resulta eficaz en casos de emociones disruptivas, no así para las emociones de largo plazo (Collins, 1990).

Leer fue una práctica distractora frente a la desesperación por los tiempos de espera institucionales. La lectura contribuyó a entender algunas situaciones de la enfermedad y a evadir la angustia frente a la expectativa de muerte del enfermo. Dormir fue un recurso frente a la tristeza de ver al familiar padecer o frente a su muerte, también ante la tensión por la situación económica, la preocupación del hacer cotidiano en el cuidado y la desesperación de no poder hacer más por el enfermo. Dormir fue un medio para suspender la situación vivida. Representó distancia de la situación emocional para continuar después con las responsabilidades del cuidado y la solución de la situación emergente. En el estudio de Murphy et al. (2006) dormir fue reportado como un escape transitorio de las responsabilidades del cuidado. En cambio, en este estudio fue una práctica reparadora para reincorporarse con otra visión a la situación del cuidado, además de un recurso para evadir por un momento la emoción sentida.

Trabajar permitió a los cuidadores olvidarse momentáneamente de la situación del cuidado, de la inquietud y la preocupación de saber si su familiar estaba bien atendido por quien lo apoyaba en esos instantes o del enojo por la actitud del enfermo hacia el cuidador. Centrar la atención en una circunstancia diferente al cuidado evitó que los cuidadores entraran en contacto con emociones que no les eran gratas. Los cuidadores comentaron que cuidaban y atendían a otras personas para evadir el dolor de la muerte de quien fuera anteriormente el receptor del cuidado. Esta necesidad de estar ocupados para no pensar en la ausencia de su familiar fue reportada por Grbich, Parker y Maddocks (2001). Los cuidadores también mencionaron ingerir antidepresivos o ansiolíticos para impedir que afloraran emociones y malestares. La manifestación corporal de las emociones tuvo prioridad en este tipo de manejo farmacológico. Los cuidadores estaban familiarizados con esta práctica a causa del conocimiento adquirido en su trabajo de cuidado. En Calasanti y King (2007) los cuidadores también optaron por esta práctica para hacer frente a los sentimientos asociados al sufrimiento.

La cuarta función del manejo emocional fue canalizar las emociones en acciones de cuidado hacia el familiar enfermo. Los cuidadores encauzaron la 
preocupación, la tristeza, la culpa y el miedo al acompañar al enfermo, al estar al pendiente de sus necesidades, al compensarlo, aconsejarlo y motivarlo. El despliegue de las prácticas asociadas con el cuidado fue documentado por Stalberg, Ekerwald y Hultman (2004) y Calasanti y King (2007). El bienestar del otro, en este caso del familiar enfermo, está vinculado con el bienestar del cuidador. Los cuidadores se mantuvieron cerca durante los procesos de atención médica de su familiar para resolver sus emociones de miedo, tristeza, preocupación e incertidumbre. Al observar los procedimientos y la evolución de la enfermedad se sentían más seguros y tranquilos respecto del porvenir de su familiar. Compensar al enfermo fue una actividad que contribuyó para que los cuidadores resolvieran sus emociones de culpa y remordimiento por sentir enojo $u$ odio hacia el enfermo o por presentar conductas no matizadas: discutir, regañar e incluso expresar verbalmente las emociones y pensamientos. Los cuidadores asumieron acciones de cuidado para

canalizar su culpa: compensaron al enfermo con más dedicación de tiempo, hicieron las cosas que le gustaban, le compraron lo que apetecía, aunque esto fuese una restricción médica - alimento prohibido- por la salud del enfermo.

Motivar y aconsejar al enfermo permitió a los cuidadores canalizar la tristeza producida por los síntomas y vivencias del familiar en su proceso de enfermedad. Los cuidadores manifestaron emociones asociadas con la falta de reconocimiento de su trabajo, lo que atribuyeron a la situación de desánimo ante la enfermedad y el tratamiento del familiar enfermo. En este sentido los cuidadores lo aconsejaron, le dieron ánimos y le sugirieron que no desesperara puesto que las cosas "saldrían bien". Procuraron brindar al enfermo un panorama más alentador respecto de su enfermedad y su futuro, y al verlo con más energía los cuidadores obtuvieron bienestar emocional. Estos hallazgos fueron reportados por Stalberg, Ekerwald y Hultman (2004) y Calasanti y King (2007).

Liberar la emoción fue la quinta función básica. Los cuidadores se procuraron sensaciones de bienestar para no sentir las emociones negativas propias de este proceso con la misma intensidad. Hacer el aseo, por ejemplo, ayudó a asimilar los acontecimientos y a sacar la emoción a través del trabajo manual de la limpieza. Fue una estrategia vinculada al género de los cuidadores, pues las mujeres lo refirieron principalmente. Esto evidencia la reproducción social de los roles según el género y cómo las personas aprenden a utilizar elementos de la vida cotidiana para favorecer su bienestar. Otra de las regulaciones mencionadas fue llorar a solas, lo que les ayudó a desahogar sus emociones. Los familiares cuidadores alcanzaron la liberación de sus emociones - coraje, molestia, enojo, desesperación, cansancio, impotencia o tristeza- mediante la estrategia de dar a conocer a los enfermos sus emociones o lo que pensaban de ellos y de su actitud. En el estudio de Gomes (2007) los cuidadores comentaron que verbalizar su sentimiento o lo que les incomodaba derivó en una sensación de alivio.

Los cuidadores también utilizaron una emoción para regular otra. La diada que caracterizó este tipo de regulación emocional fue tristeza-enojo. Decidieron disminuir la tristeza y liberarla en forma de enojo, lo que les permitió soslayar el llanto, además de que fue el medio de contención para salir airosos ante el observador, ya fuese el enfermo u otro familiar. Platicar con alguien permitió la expresión emocional de forma parcial: ayudó a los cuidadores a manifestarla, aunque no fue en el momento vívido de la emoción. Como menciona Kilburn (1996), conversar con otro redundó en un intercambio de conocimientos y experiencias para sobrellevar el cuidado cotidiano. Para que los cuidadores pudieran liberar sus emociones fue determinante contar con una red social de apoyo, ya fuese a fin de resolver cuestiones prácticas del cuidado y de los servicios de atención o para solventar los gastos del tratamiento o para tener con quién compartir experiencias y emociones.

La sexta función básica fue parcializar, entendida como la omisión de una parte del contexto que producía la emoción dolorosa. Para lograrlo, los 
cuidadores centraron su atención en un punto neutro de la situación emocional, lo que les permitió no estar preocupados, omitir cualquier emoción relacionada con las circunstancias futuras del enfermo y enfocarse en las actividades concretas del cuidado. Parcializar se relacionó directamente con los procesos cognitivos de los cuidadores, que omitieron el nombre de la enfermedad - cáncerde su familiar o la posibilidad de muerte del enfermo en fase terminal. Acacia fue la única cuidadora que reconoció la estrategia que utilizaba, ayudada por su proceso psicoterapéutico. Comentó:

Cuando apunté "para apoyo de los enfermos con cáncer", yo -y como dice la psicóloga: "ha sido parte de tu organismo muy sabio" - siento como que no es cáncer, ¿sí me entiendes? Como que es una enfermedad muy seria pero no le pongo nombre y aparte como que no me la quiero creer mucho (Acacia, 2009).

La séptima función básica fue el cambio y tuvo un objetivo doble. Por un lado, cambiar la situación emocional. Los cuidadores se centraron en la transformación del elemento social para operar el cambio de la emoción sentida. Una de las maneras de conseguirlo fue la expresión de la emoción o del pensamiento no guiada por el enojo disruptivo, sino motivada por la necesidad de hablar directamente con el interlocutor y manifestar lo que pensaban y esperaban de él. Esta estrategia procuró el cambio de la situación emocional, aunque éste estuvo sujeto a las decisiones y actitudes del otro, como respuesta a la expresión del cuidador. Las redes sociales también participaron en el cambio de la situación o de las emociones de los cuidadores. Por lo regular, quien tuvo influencia en el cuidador y en sus emociones fue una persona de confianza y de jerarquía social, espiritual o emocional para él, de modo que fue escuchada. Los familiares y amigos ayudaron a reducir el sufrimiento, la desesperación y la preocupación al proporcionar a los cuidadores apoyo económico y humano en el trabajo del cuidado. Las redes formales de las instituciones —Cáritas y Sistema para el Desarrollo Integral de la Familia (DIF) - brindaron principalmente respaldo económico, lo que alivió la preocupación, la tensión y la presión relacionadas con la falta de recursos económicos.

Por otra parte, asumir el rol de autoridad - cumplir la sentencia o mandato que el cuidador empleó para la solución de la situación emocional- favoreció el cambio de actitud del enfermo y permitió el cese de la emoción en el cuidador. Pedir a Dios como estrategia de cambio tuvo como característica el acercamiento espiritual del cuidador a Dios por medio de la lectura de la Biblia y de hacer oración. Inmediatamente después, enunciaron como "inspiración divina" las acciones necesarias para modificar las condiciones que generaron la emoción. La toma de decisiones a través de la oración es un dato que coincidió con los proporcionados por Richards et al. (2003), que afirman que sus informantes transformaron sus emociones y pudieron sentirse acompañados por esta vía.

Poner límites fue la estrategia que permitió al cuidador conservar su espacio personal y salvaguardar sus emociones. Uno de ellos fue el límite de tiempo, desarrollado en las situaciones en que los cuidadores se declararon incapaces de realizar más actividades de las que ya desarrollaban y en las que sentían presión, frustración, intranquilidad y pendiente. La acción específica fue poner límites en los tiempos de cuidado y ceder la responsabilidad a otra persona. Los cuidadores recurrieron al límite emocional cuando llegaron a considerar que su trabajo era inútil o cuando persistía la renuencia del enfermo ante el tratamiento o el cuidado, de modo que los cuidadores decidieron separar sus emociones de las acciones de cuidado al no poder deslindarse del estado de salud de su pariente de manera definitiva debido a su principio del deber ser como buen familiar y buen cuidador. Calasanti y King (2007) mencionan un proceso similar en su estudio al que llamaron "bloquear las emociones".

La ayuda social o profesional orientada al familiar fue básicamente para atender situaciones fuera del alcance de los cuidadores, debido a su ignorancia en 
el manejo de los síntomas o de la enfermedad, lo que les generó tristeza, susto, impacto emocional y desesperación. La ayuda funcionaba cuando presentaban conflicto emocional respecto de la posible pérdida de su familiar enfermo, y en los casos en que sintieron coraje, estrés, frustración y presión relacionados con el trabajo de cuidado.

El cambio se refiere también a la valoración emocional en las estructuras internas para intervenir en la emoción sentida. Ello se logró mediante el conocimiento y análisis de la situación emocional y de la propia enfermedad para discriminar entre los momentos característicos del padecimiento, los de crisis y los que requieren de atención clínica inmediata. Por otro lado, se echó mano de recursos cognitivos y culturales para procesar la situación emocional. Los cuidadores realizaron actividades como escribir cartas o historias ante la preocupación por las demandas del trabajo cotidiano, luego de lo cual estuvieron en condiciones de procesar y resolver sus emociones. Hablar consigo mismo para motivarse, corregirse o controlar su respuesta emocional se relacionó particularmente con las emociones de estrés, presión, nervios, responsabilidad, preocupación y depresión vinculadas a la situación económica, relacional y del hacer cotidiano. Las frases que surgieron respecto de esto fueron: "No gano nada con preocuparme", "Esta situación va a cambiar", "Ya párale a tus nervios", "No es tu responsabilidad”, “Para qué me estreso?", “Tengo que ser amable”. Poindexter (2001) identificó que los cuidadores utilizaron esta estrategia ante las crisis en el manejo de los enfermos ViH positivos. Ellos, al igual que los cuidadores en este estudio, procuraron motivarse para continuar el cuidado, libres de preocupaciones, y sobrellevar los momentos críticos.

Comparar la situación emocional con la de otros hizo posible que los cuidadores revaloraran su salud, la de sus familiares enfermos y su trabajo como cuidadores. La estrategia se llevó a cabo frente a emociones como depresión, preocupación y dificultad ante la situación del cuidado y de la enfermedad. Stalberg, Ekerwald y Hultman (2004) registraron este tipo de estrategia, pero en relación con la necesidad emocional de normalizar la situación de enfermedad mental del familiar enfermo. En este estudio la finalidad fue otra: se comparó con situaciones de vida de otras personas en una condición menos favorable socialmente o de salud del enfermo. De manera que cuando el cuidador volvía la mirada a su circunstancia la percibía menos adversa y más fácil de sobrellevar. El proceso fue observar la situación ventajosa que había sido invisibilizada por la cercanía y cotidianidad de su trabajo. Los cuidadores justificaron diversas situaciones dentro del cuidado: deslindar al enfermo de responsabilidad por sus actitudes - lo que fortaleció la continuidad de la cercanía afectiva-, disminuir su culpa después de expresarle enojo al argumentar la imperfección humana - "no somos personas perfectas, todos nos desesperamos"- y el nuevo rol como parte del destino personal.

El proceso para apropiarse de una idea relacionada con la situación emocional fue escuchar la respuesta de otro a la pregunta o pensamiento que aquejaba al cuidador y que no había exteriorizado con nadie. Al apropiarse del mensaje necesitado el cuidador pudo resolver su emoción, sentir tranquilidad, disipar su culpa y responsabilidad y además aclarar la situación emocional. Los cuidadores también recurrieron a Dios para cambiar sus emociones. El acercamiento a Dios fue distinto según las fases de la enfermedad del familiar. Ante la noticia del diagnóstico y de los primeros síntomas surgió el reclamo, pues les pareció poco justo. Después pidieron a Dios y suplicaron ayuda para aliviar a su familiar, para resolver sus emociones y para sobrellevar el cuidado. Por último, agradecieron a Dios la destreza que adquirieron en el cuidado y la salud del enfermo o incluso por su muerte.

No hubo distinción de nivel socioeconómico o enfermedad para acudir a Dios y solicitarle ayuda. Los cuidadores dijeron sentirse acompañados, tranquilos y con la capacidad para aceptar los acontecimientos y dejar de sentir las emociones no gratas. "Pedir a mis muertos" fue una estrategia de los cuidadores ante la tristeza de haber perdido a su familiar enfermo y 
frente a la dificultad de una nueva situación que resolver, por lo que pidieron ayuda en la toma de decisiones certeras. Para los cuidadores representó una manera de mantener la relación con su familiar y de sentirse tranquilos después de la muerte del enfermo al saberlo en un lugar sin dolor. Además, significó saberse protegidos, tal como los enfermos cuando los cuidadores los asistieron. Otras dos estrategias para procesar la carga emocional y lograr el cambio de las valoraciones emocionales fueron imaginar y usar un objeto sustituto. El ejercicio de imaginar se llevó a cabo ante la desesperación, el estrés, el enojo, las ganas de abandonar el cuidado del enfermo y sentirse sin libertad para desarrollar las actividades personales. La frase central fue "me gustaría", a la que le siguieron relatos que expresaron la recreación imaginativa de lo que podía ser. En las narraciones se observó la necesidad de un sentido de vida personal alejado del cuidado, de ayuda para sí mismos y de libertad de hacer. El uso de un objeto sustituto se vinculó con las emociones de dolor, tristeza, pérdida, soledad y ausencia, producto de la muerte del familiar enfermo. Los cuidadores recurrieron a los objetos, espacios o personas que les hicieran sentir la cercanía con su familiar ausente.

Las características particulares de las estrategias de regulación emocional de función básica o primaria radican en que son aplicables a otras situaciones emocionales aparte de las del cuidado y procuran dar solución de forma inmediata, mediata y de largo plazo, además de que ilustran sobre el involucramiento de los procesos subjetivos, intersubjetivos y socioculturales por los que pasan los cuidadores para lograr regular sus emociones, lo que demuestra la sabiduría y capacidad de los cuidadores para el manejo de sus emociones y de las situaciones emocionales a las que se enfrentan en su trabajo cotidiano del cuidado.

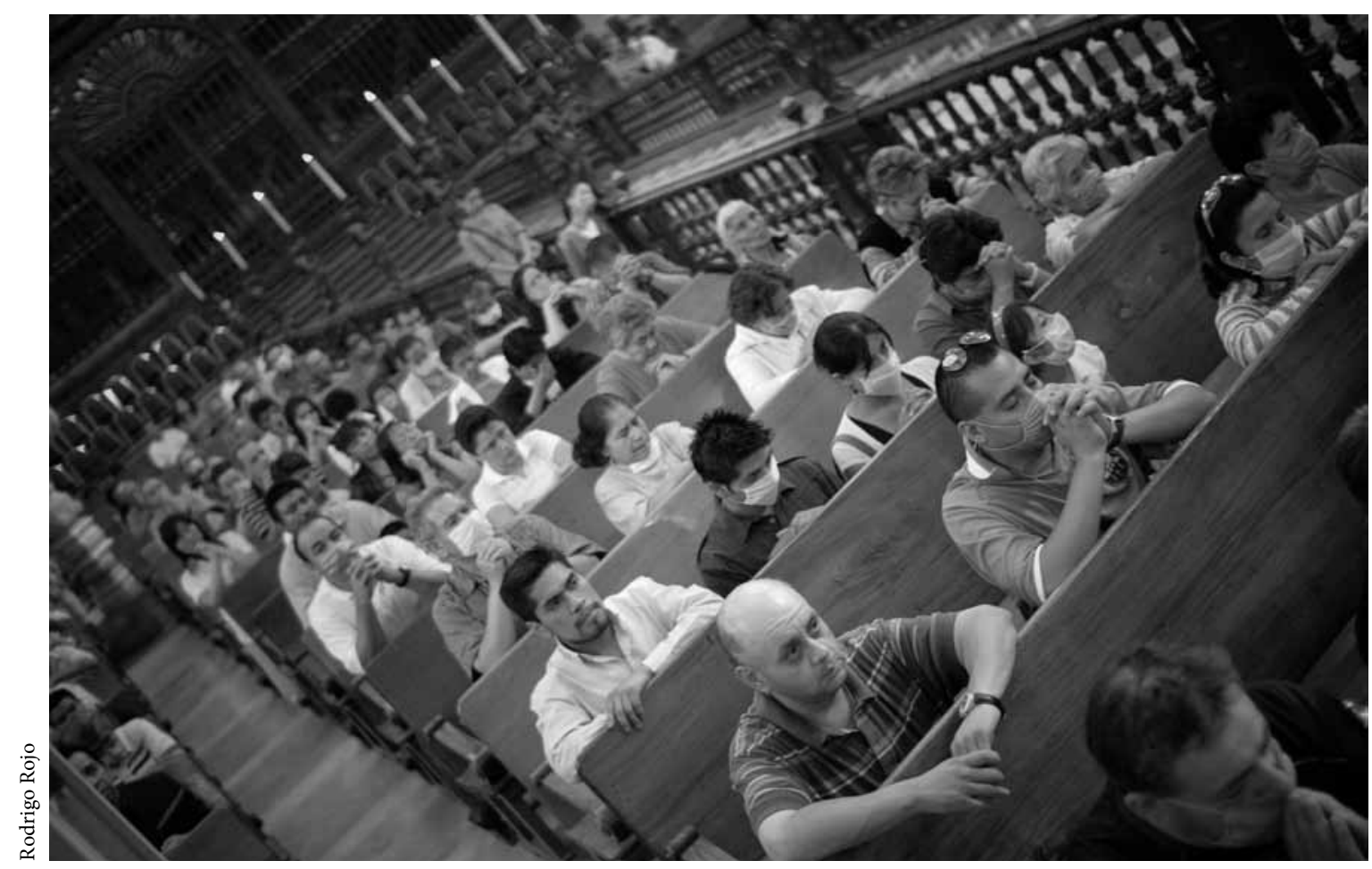

Misa en la Catedral Metropolitana para orar por el fin de la epidemia de influenza, 2009. 


\section{Estrategias de regulación emocional y la búsqueda del bienestar}

Las funciones de segundo orden respondieron de manera directa a las finalidades del cuidado (Fisher y Tronto, 1990; Engster, 2005), regidas bajo el principio de bienestar de acuerdo con la teoría feminista de la ética del cuidado (Ungerson, 1983; Noddings, 1984; Engster, 2005). Las estrategias de regulación emocional se orientaron a tres instancias: 1) la subjetividad relacionada con el bienestar del cuidador -cuidado dirigido a los otros y a uno mismo (Engster, 2005)—, la protección de la persona y su integridad emocional; 2) el enfermo de cáncer o diabetes, su bienestar, protección del dolor emocional y satisfacción de sus necesidades (Ungerson, 1983; Engster, 2005), y 3 ) la permanencia subjetiva e intersubjetiva en la sociedad, la lucha del cuidador para lograr su subsistencia en el mundo social (Gilligan, 1995).

Entre las funciones orientadas a la subjetividad se ubica la protección de la integridad personal del cuidador a nivel social e individual. En el primer caso, los cuidadores procuraron contener las emociones y simularlas para mantener la imagen personal de fortaleza ante los demás, prefirieron ser discretos y conservar las normas sociales de expresión emocional (Hochschild, 1990). A su vez, los cuidadores optaron por protegerse del dolor que les causó alguna emoción, como la tristeza, la desesperación, la angustia, el sufrimiento, el resentimiento y la frustración en relación con el sufrimiento de su familiar y por las dificultades en el cuidado, por lo que desplegaron acciones de regulación emocional vinculadas con las siete funciones básicas. La recuperación emocional del cuidador se dirigió hacia su fortaleza emocional para continuar el trabajo de cuidado y recuperar el sentido de vida perdido durante su trayectoria como cuidador.

Recuperar la fortaleza emocional, desde la perspectiva de los cuidadores, tiene que ver con estrategias que les ayudaron a evadir, canalizar, liberar, parcializar o cambiar sus emociones y tener la capacidad de hacer frente a las adversidades del cuidado y salir librado lo más airoso posible del dolor emocional. Cuando los cuidadores sintieron perdido el sentido de su vida, implementaron estrategias, con una eficacia mayor a la esperada, para evadir y canalizar sus emociones. Los cuidadores centraron su atención en proyectos nuevos, amores o situaciones de interés, lo que les proporcionó la ilusión y la motivación de tener una meta adicional a la de cuidar de su familiar enfermo.

El crecimiento personal del cuidador fue logrado mediante el autoconocimiento y el desarrollo personal. La estrategia que facilitó este proceso fue pedir ayuda profesional y favorecer estrategias de cambio. Consistió en retomar su vivencia como desarrollo personal, académico o social, que permitiera la visualización de su experiencia y el entendimiento del cuidado de otras personas. Sacar la emoción contenida fue otra de las funciones secundarias en aras de la subjetividad. Los cuidadores mencionaron principalmente las estrategias relacionadas con liberar, hacer el aseo, llorar, platicar con alguien y pedir a Dios, acciones que les ayudaron a pensar la situación emocional con menos trascendencia, sentirse a gusto y tranquilos. Cuando estas formas de regulación emocional no fueron suficientes para externar la emoción contenida, los cuidadores hicieron referencia al cuerpo como una entidad sabia con capacidad de expulsar la emoción a través de la enfermedad y de los malestares corporales.

Respecto de las funciones enfocadas a la otredad, las estrategias de regulación emocional fueron proteger al enfermo del dolor emocional y resolver las necesidades del cuidado. Las acciones para contener, simular, evadir, liberar, cambiar y, principalmente, canalizar tuvieron por objetivo proteger al otro del dolor emocional de las situaciones familiares, sociales, de enfermedad y de las emociones del cuidador. Contener y simular la emoción y cambiar la valoración emocional o la situación reportaron al cuidador la capacidad de solucionar las necesidades emergentes del cuidado, ya fuesen los trámites de la atención médica o el trabajo del cuidado. 
Las funciones orientadas a la permanencia social del cuidador se encaminaron a preservar su relación con el enfermo y con los otros, para lo cual los cuidadores procuraron estar libres de conflictos con los otros tanto como fuese posible. Otra forma fue intentar hacer "reaccionar" al otro, objetivo en función del cual los cuidadores desplegaron estrategias de cambio de la situación, sobre todo por medio de la expresión de sus emociones o pensamientos a los otros, para conmover o confrontar. El objeto de cambio fueron principalmente las actitudes del enfermo o de algún miembro de la familia con vistas a su participación en el cuidado.

En síntesis, a lo largo de este apartado se observa la relación existente entre las funciones de nivel primario y las de segundo nivel. Para el logro de una función secundaria se requiere de la activación de dos o más funciones de primer nivel. Es necesario recapitular que tanto las emociones, las estrategias de regulación emocional y el cuidado son fenómenos y construcciones sociales. Desde ahí es posible explicar su naturaleza y complejidad. En la teoría feminista de la ética del cuidado y en la teoría construccionista de la sociología de las emociones se reconoce, por una parte, la función del cuidado como la actividad encaminada a proteger al otro, a uno mismo y a su mundo. En este sentido, considerando ambas posturas, el manejo de las emociones de los cuidadores es una forma de cuidado en aras de su propia protección y de la del otro, así como de su permanencia en el mundo social (Fischer y Tronto, 1990; Gordon, 1990). Es decir, su existencia recae en las actividades de reproducción social que permiten la integración de las personas en diferentes escenarios sociales y su continuidad en el mundo de la vida cotidiana.

\section{CONCLUSIONES}

Uno de los aspectos más sobresalientes del grupo socioeconómico para el despliegue de una estrategia determinada para la regulación emocional fue la escolaridad del cuidador. Entre los cuidadores de escolaridad primaria completa o no concluida se observó que los procesos mentales relacionados con la elaboración intelectual de la situación emocional fueron más limitados que entre los cuidadores con un nivel superior de escolaridad. Esto quedó de manifiesto en los procesos de escritura, análisis, organización y construcción de ideas a partir de la elaboración de cartas, cuentos y diagramas para resolver su estado emocional. Es evidente que los procesos de escolarización brindan herramientas sociales y cognitivas para la solución de la situación emergente del cuidado (CEPAL, 2009) y para el manejo de las emociones.

Pertenecer a un estrato socioeconómico u otro no representó una diferencia entre los cuidadores respecto de la devoción para pedir a Dios por la solución de la situación o por la transformación de sus emociones. De manera general, los significados espirituales, gestados antaño en la cultura mexicana, sirvieron para hacer frente a las emociones de los cuidadores y ayudaron en la comprensión de los procesos de enfermedad, de limitación personal y de la muerte del enfermo (Vázquez, 2008). En cuanto al tipo de enfermedad, las diferencias estuvieron marcadas por las emociones a las que se dio solución, surgidas según la situación de la enfermedad: la estrategia referida por los cuidadores de enfermos de cáncer fue parcializar, lo que no ocurre en los casos de diabetes, pues prefirieron omitir el nombre del padecimiento para disminuir el significado de pérdida y la posibilidad de muerte que les significaba.

La capacidad de desarrollar las estrategias de regulación emocional es socialmente construida y motivada por medio de la interacción de la persona con su mundo, es decir, del aprendizaje social en el que el cuidador - a partir de su historia de vida y de la educación provista en la familia y en la sociedad en general- adquiere la capacidad para llevar a cabo una forma de regulación específica. Los cuidadores exhibieron una gama de estrategias para la regulación de emociones poco gratas que de acuerdo con su cultura 
era mejor no manifestar abiertamente, como la tristeza, el rencor o el enojo. Esta diversidad no se observó en el caso de las emociones agradables, como la alegría, el bienestar o el orgullo. Cuando se les cuestionó sobre qué hacían cuando se sentían orgullosos o alegres, los cuidadores no refirieron acciones de regulación dirigidas a estas emociones y se limitaron a mencionar la asociación con emociones de la misma naturaleza: "me siento alegre", por ejemplo, o respondieron con algún calificativo que describiera su estado emocional, como "sentir bonito". Los aspectos culturales manifestados a través de las normas sociales de regulación emocional, aunados al deber ser del cuidado, las creencias y las costumbres de los cuidadores, fueron elementos sociales que influyeron de forma determinante sobreponiéndose a la situación socioeconómica del cuidador y a la enfermedad de su familiar.

Una de las principales aportaciones de este trabajo de investigación fue enriquecer el modelo del manejo emocional de Hochschild (1990) con el desarrollo de una clasificación de doble funcionalidad en las acciones del manejo emocional, donde la función primaria se encamina a la resolución de la emoción o situación emocional y las funciones de segundo nivel atienden el principio de bienestar. En este trabajo también se sientan las bases para entender que la regulación emocional no sólo cumple una función subjetiva de bienestar emocional, sino que persigue la perpetuidad de las relaciones sociales y responde al principio de cuidado, en el que se procura el bienestar social y la continuidad de las personas en su entorno físico y cultural.

En el funcionamiento de las estrategias de regulación emocional se advierte la retroalimentación entre lo subjetivo y lo cultural, sobre todo en la actuación de los cuidadores según los significados culturales y en el empleo de su bagaje cultural para desplegar conductas aceptadas socialmente. Mediante el manejo emocional, los cuidadores contribuyeron a las relaciones simbólicas en los diferentes momentos del proceso del cuidado. Sujeto y sociedad se construyen y transforman mutuamente. Una posible limitación del trabajo es que se concentró en recuperar la perspectiva de los cuidadores y no la de los enfermos o la de otros familiares involucrados en las tareas del cuidado. Si bien se logró dar respuesta a la pregunta de investigación, queda pendiente - como línea de investigación posible - un análisis social más amplio que explique con un mayor nivel de complejidad el objeto de estudio y que explicite con fuerza la dimensión sociocultural, ética y política del cuidado y las formas en que las emociones sociales mantienen, reproducen o trastocan y transforman un orden social que ha depositado en el sujeto - en este caso en el cuidador familiar- responsabilidades que lo sobrepasan y que ponen de manifiesto la necesidad de construir un pacto social basado en la complementariedad en relación con el cuidado y desde el marco de los derechos sociales, y por tanto desde la perspectiva de la equidad y la justicia social.

El cuidado es un problema central en la agenda pública para América Latina. Las cargas que representa crecerán exponencialmente en las próximas décadas (CEPAL, 2009), según las proyecciones demográficas para la región. Simultáneamente, los procesos de precarización del cuidado y de la existencia misma (Vara, 2006; Enríquez, 2011) confrontan las posibilidades reales de los sujetos, principalmente de las familias y sobre todo de las mujeres, para hacer frente a un problema social que los rebasa y que deslinda a las instituciones, al mercado y a la sociedad en general de una responsabilidad que compete a todos y que puede asumirse sólo de esa manera.

El cuidado, en tanto objeto de estudio interdisciplinar, requiere de acercamientos teórico-metodológicos que deriven en la articulación de conceptos y dimensiones provenientes de espacios entre y más allá de disciplinas, como la psicología, la antropología, la sociología y la medicina. Las emociones sociales ancladas en narrativas múltiples y vinculadas a prácticas específicas en torno al cuidado requieren ser analizadas a partir de enfoques socioculturales que develen las formas complejas en que se ha interiorizado e individualizado una labor 
que compete a la sociedad entera. El cuidado, así como el bienestar, son eminentemente sociales y la colectivización de los mismos es una tarea central en nuestro país que exige de pasos firmes que permitan anticiparnos a un proceso de envejecimiento que demandará cada vez más cargas mayores de cuidado. Adquiere centralidad pensar y construir a partir de la noción de "integralidad en el cuidado". La provisión del cuidado es una cuestión ética y política, un derecho de todo ser humano, que urge a poner en práctica acciones colectivas y públicas (Pinheiro y Araújo de Mattos, 2008 a y b, 2009; Pinheiro y Cohelo Lopes, 2010).

\section{Bibliografía}

Calasanti, Toni y King Neal, 2007, “Taking 'Women's Work' 'Like a Man': Husbands' Experiences of Care Work', en The Gerontologist, vol. 47, núm. 4, pp. 516-527.

Collins, Randall, 1990, "Stratification, Emotional Energy and the Transient Emotions", en Theodore Kemper (ed.), Research Agendas in the Sociology of Emotions, State University of New York Press, Nueva York, pp. 27-57.

Comisión Económica para América Latina y el Caribe (CEPAL), 2009, Panorama social de América Latina, Comisión Económica para América Latina y el Caribe, Santiago de Chile.

Engster, Daniel, 2005, "Rethinking Care Theory: the Practice of Caring and the Obligation to Care", en Hypatia, vol. 20, pp. 50-74.

Enríquez, Rocío, 2008, El crisol de la pobreza: mujeres, subjetividades, emociones y redes sociales, Instituto Tecnológico y de Estudios Superiores de Occidente, Guadalajara, México.

_ _ 2011, "La construcción social del cuidado y el bienestar social en México: una aproximación a los debates contemporáneos", ponencia, XII Seminario Nacional de Política Social en México, Universidad Iberoamericana, México.

Fisher, Berenice y Joan Tronto , 1990, “Toward a Feminist Theory of Caring", en Emily Abel y Margaret Nelson (eds.), Circles of Care Work and Identity in Women's Lives, State University of New York Press, Nueva York, pp. 35-62.
Gilligan, Carol, 1995, "Moral Orientation and Moral Development", en Virginia Held (ed.), Justice and Care. Essential Readings in Feminist Ethics, Westview Press, Colorado, pp. 31-46.

Gomes, Ana, Aires Consuelo y Bessa Maria, 2007, "O fazer do cuidador familiar: significados e crenças", en Revista Brasileira de Enfermagem, vol. 60, núm. 5, pp. 530-534.

Gordon, Steven, 1990, "Social Structural Effects on Emotions", en Theodore Kemper (ed.), Research Agendas in the Sociology of Emotions, State University of New York Press, Nueva York, pp. 145-179.

Graham, Hilary, 1983, "Caring: A Labour of Love”, en Janet Finch y Dulcie Groves (eds.), A Labour of Love, Routledge and Kegan Paul, Londres, pp. 13-30.

Grbich, Carol, Deborah Parker y Ian Maddocks, 2001, "The Emotions and Coping Strategies of Caregivers of Family Members with a Terminal Cancer", en Journal of Palliative Care, vol. 17, núm. 1, pp. 30-36.

Hammersley, Martyn y Paul Atkinson, 1994, Etnografía. Métodos de investigación, Paidós, Barcelona.

Hochschild, Arlie, 1990, "Ideology and Emotion Management: A Perspective and Path for Future Research", en Theodore Kemper (ed.), Research Agendas in the Sociology of Emotions, State University of New York Press, Nueva York, pp.117-144.

Instituto Nacional de Estadística, Geografía e Informática (INEGI), 1990, XI Censo General de Población y Vivienda 1990, datos por área de geoestadística básica urbana en Jalisco, Instituto Nacional de Estadística, Geografía e Informática, México.

_ 1994, "Jalisco. Resultados definitivos. Datos por AgeB urbana", en XI Censo General de Población y Vivienda 1990, Instituto Nacional de Estadística, Geografía e Informática, México.

Kilburn, John, 1996, "Network Effects in Caregiver to Care-recipient Violence: A Study of Caregivers to those Diagnosed with Alzheimer's Disease", en Journal of Elder Abuse \& Neglect, vol. 8, núm. 1, pp. 69-80.

Krippendorff, Klaus, 1990, Metodología de análisis de contenido, Teoría y práctica, Paidós, Barcelona.

Le Breton, David, 1998, Las pasiones ordinarias. Antropología de las emociones, Ediciones Nueva Visión, Buenos Aires.

Murphy, Nancy et al., 2006, "The Health of Caregivers for Children with Disabilities: Caregiver Perspectives", en Child: Care, Health and Development, vol. 33, núm. 2, pp. 180-187.

Noddings, Nel, 1984, Caring. A Femenine Approach to Ethics and Moral Education, University of California Press, Berkeley. 
Pautassi, Laura, 2007, El cuidado como cuestión social desde un enfoque de derechos, Comisión Económica para América Latina y el Caribe, Chile.

Pinheiro, Roseni, 2008, Cuidado as fronteiras da integralidade, Instituto de Medicina Social, Universidade do Estado do Rio de Janeiro, Centro de Estudos e Pesquisa em Saúde Colectiva, Associaçao Brasileira de Pos-graduaçao em Saúde Coletiva, Brasil.

$\longrightarrow, 2009$, Razões públicas para a integralidade em saúde: o cuidado como valor, Centro de Estudos e Pesquisa em Saúde Colectiva, Instituto de Medicina Social, Universidade do Estado do Rio de Janeiro, Associaçao Brasileira de Pos-graduaçao em Saúde Coletiva, Laboratório de Pesquisas sobre Práticas de Integralidade em Saúde, Brasil.

- y Ruben Araújo de Mattos (comps.), 2008, Cuidar do Cuidado: Responsabilidade com a Integralidade das Ações de Saúde, Centro de Estudos e Pesquisa em Saúde Coletiva, Instituto de Medicina Social, Universidade do Estado do Rio de Janeiro, Associaçao Brasileira de Posgraduaçao em Saúde Coletiva, Laboratório de Pesquisas sobre Práticas de Integralidade em Saúde, Brasil.

_- y Tatiana Coelho Lopes (comps.), 2010, Ética, técnica e formação: as razões do cuidado como direito a saúde, Centro de Estudos e Pesquisa em Saúde Coletiva, Instituto de Medicina Social, Universidade do Estado do Rio de Janeiro, Associaçao Brasileira de Pos-graduaçao em Saúde Coletiva, Laboratório de Pesquisas sobre Práticas de Integralidade em Saúde, Brasil.

Poindexter, Cynthia, 2001, “'I'm Still Blessed': The Assets and Needs of HIV-affected Caregivers over 50", en Families in Society, vol. 85, núm. 5, pp. 525-536.

Pujadas, Joan, 2000, "El método biográfico y los géneros de la memoria”, en Revista de Antropología Social, núm. 9, pp. 127-158.
Richards, Anne et al., 2003, "Subjective Experiences of Prayer among Women Who Care for Children with HIV", en Journal of Religion and Health, vol. 42, núm. 3, pp. 201-219.

Robles, Leticia, 2007, La invisibilidad del cuidado a los enfermos crónicos. Un estudio cualitativo en el barrio de Oblatos, Editorial Universitaria, Universidad de Guadalajara, Guadalajara.

Stalberg, Gabriella, Hedving Ekerwald y Christina Hultman, 2004, "At Issue: Sibling of Patients with Schizophrenia: Sibling Bond, Coping Patterns, and Fear of Possible Schizophrenia Heredity", en Schizophrenia Bulletin, vol. 30, núm. 2, pp. 445-458.

Strauss, Anselm y Juliet Corbin, 2008, Pesquisa qualitativa. Técnicas e procedimentos para o desenvolvimento de teoria fundamentada. Métodos de pesquisa, Artmed, Brasil.

Tronto, Joan, 1993, A Political Argument for an Ethic of Care, Routledge, Nueva York.

Ungerson, Clare, 1983, “Why do Women Care?”, en Janet Finch y Dulcie Groves (eds.), A Labour of Love, Routledge and Kegan Paul, Londres, pp. 31-49.

Vara, María de Jesús, 2006, "Precarización de la existencia y huelga de cuidados”, en María de Jesús Vara (coord.), Estudios sobre género y economía, Akal, Madrid.

Vázquez, Elba, 2008, "Las emociones en el cuidado de enfermos crónicos: revisión del estado del arte", tesis de maestría en ciencias de la salud pública, Universidad de Guadalajara, Guadalajara.

Vázquez, Felipe (comp.), 2008, Construyendo la vida a partir de la muerte, Gobierno del Estado de Veracruz, México. 